\title{
Two novel associations in a case with Walker Warburg syndrome; Enophthalmia, interhemispheric cyst and cerebral hematoma
}

\author{
Sultan Kaba ${ }^{1 *}$, Murat Doğan ${ }^{1}$, Mehmet Deniz Bulut ${ }^{2}$, Keziban Bulan $^{1}$, Nihat Demir ${ }^{1}$, Lokman \\ Üstyol ${ }^{1}$, Zeyneb Ümit Bozdoğan ${ }^{1}$, Nesrin Ceylan ${ }^{1}$ \\ ${ }^{1}$ Pediatrician, Yuzuncu Yil University, School of Medicine, Department of Pediatrics, Van, Turkey \\ ${ }^{2}$ Radiologist, Yигиnсu Yil University, School of Medicine, Department of Radiology, Van, Turkey
}

\begin{abstract}
There were severe brain malformations, hydrocephaly, myopathy and congenital cataract in a 5-month old girl presented with seizure. Walker Warburg syndrome is the most severe form of congenital muscular dystrophy accompanied by brain and eye anomalies. The findings in this case fulfilling diagnostic criteria of Walker Warburg syndrome other than type 2 lissencephaly suggest an intermediate form between Walker Warburg syndrome and muscle-eye-brain disease. In this manuscript, we intended to present this case presenting features (enophthalmia, interhemispheric cyst and cerebral hematoma) not reported previously in the spectrum of congenital muscular dystrophy-dystroglycanopathy with brain and eye anomalies.
\end{abstract}

Key Words: Walker Warburg syndrome, enophthalmia, interhemispheric cyst, cerebral hematoma

\section{Introduction}

Congenital muscular dystrophydystroglycanopathy with brain and eye anomalies (type A) has a highly variable clinical spectrum. It is a genetically heterogeneous, autosomal recessive disorder with severe mental retardation, congenital muscular dystrophy, characteristic eye and brain malformations that result in premature death. Clinical spectrum can vary from the most severe form, Walker Warburg syndrome (WWS), to a less severe form of muscle-eye-brain (MEB) disease (1). Its incidence is unknown. In MEB disease, patients can be able to walk in rare instances and speak with a few words. However, clinical presentation is more severe in WWS and the patients generally die in the first year of life. These disorders belong to a group of disease known as dystroglycanopathies caused by glycolysation defect in dystrophin-associated glycoprotein 1 DAG1 (2). In WWS, the most common clinical findings include cobblestone (type 2) lissencephaly, cerebellar malformations and retinal malformations, while presence or absence of congenital contractures, cleft lip and palate, macrocephaly or microcephaly, hypoplasia in midbrain structures, ventricular dilatation and microphthalmia are highly variable (1).
Here, we presented a case which is thought to be an intermediate form between WWS and MEB with its phenotypic features, laboratory findings and cerebral magnetic resonance imaging (MRI) features, as it displayed features that aren't reported previously including enophthalmia, interhemispheric cyst and cerebral hematoma.

\section{Case report}

A 5-months old girl was presented to outpatient clinic with seizure including shifted eyes and contractions in arms and legs. She was second child of parents with consanguinity (first degree cousins). It was found that hydrocephaly was detected on the week 20 during prenatal followup. The first child of family was healthy while the second pregnancy resulted in abortion. There was growth retardation and neuromotor retardation as well as generalized hypotonia and dysmorphic features. In the physical examination, there was bulging fontanel, frontal bossing, profoundly localized eyes, compressed nasal bridge, hook nose, micrognathia (Figure 1) and bilateral flexion contractures (involving elbow and wrist) at upper extremities. At presentation, weight was $3800 \mathrm{~g}$ $\left(<3^{\text {rd }}\right.$ percentile $)$ while height was $59 \mathrm{~cm}\left(<3^{\text {rd }}\right.$ percentile). In ophthalmological examination, 


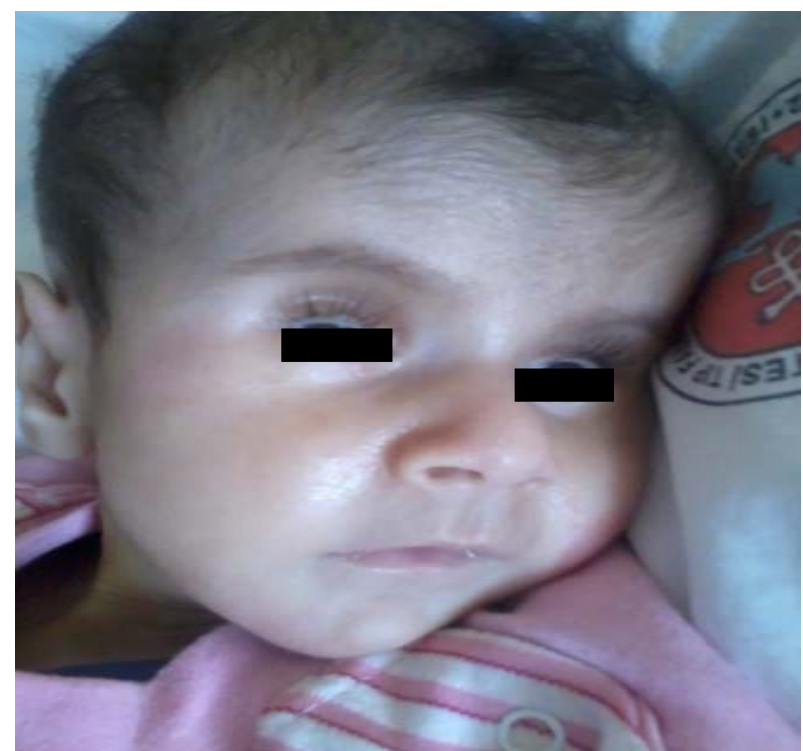

Fig. 1. Dysmorphic facial features (bulging fontanel, frontal bossing, profoundly localized eyes, compressed nasal bridge, hook nose, micrognathia) are seen.

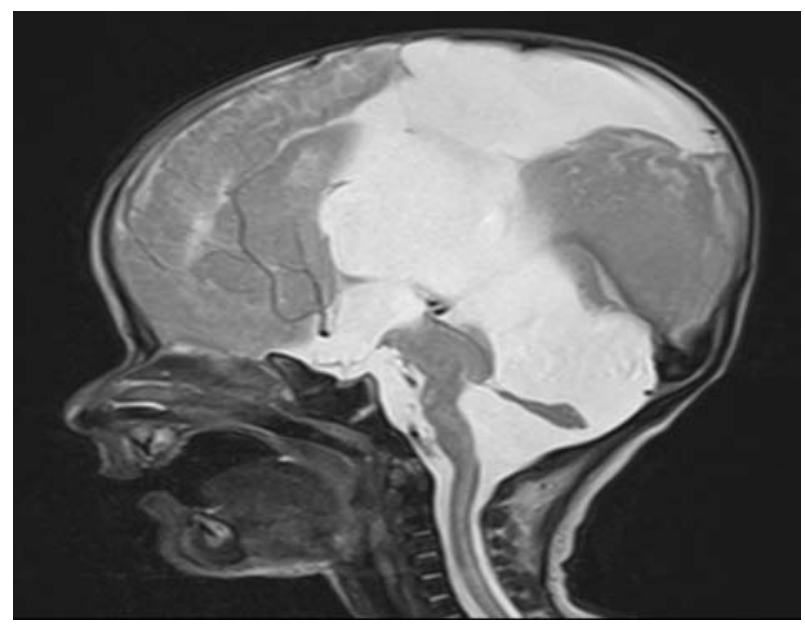

Fig. 3. T2-weighted sagittal image shows hypoplasia of cerebellum and brainstem. No corpus callosum is seen.

bilateral congenital cataract and posterior synechiae were observed while fundus examination couldn't be performed due to cataract. In laboratory, complete blood count, liver and kidney functions, serum electrolyte values, calcium, phosphorus, coagulation tests and thyroid function tests were within normal range while creatine kinase was markedly high (14037.48 $\mathrm{U} / \mathrm{L}$; age-adjusted reference range: $<228 \mathrm{U} / \mathrm{L})$. Pachygyria-agyria complex, type 1 lissencephaly and dilated occipital horns of lateral ventricles (Figure 2), hypoplasia of cerebellum and brainstem and corpus callosum agenesis (Figure 3)

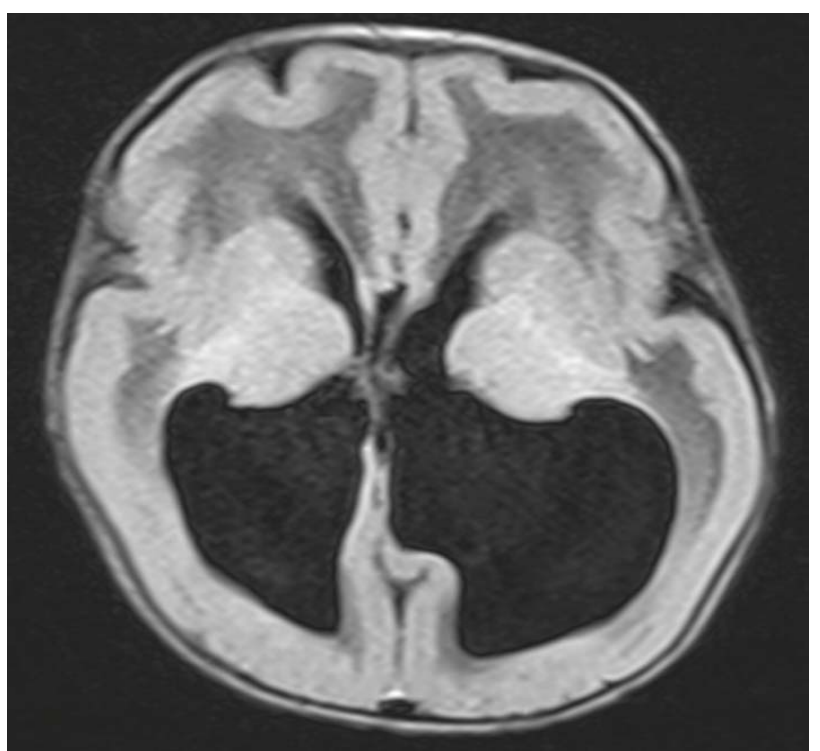

Fig. 2. Flair axial image shows type 1 lissencephaly and dilated occipital horns of bilateral ventricles.

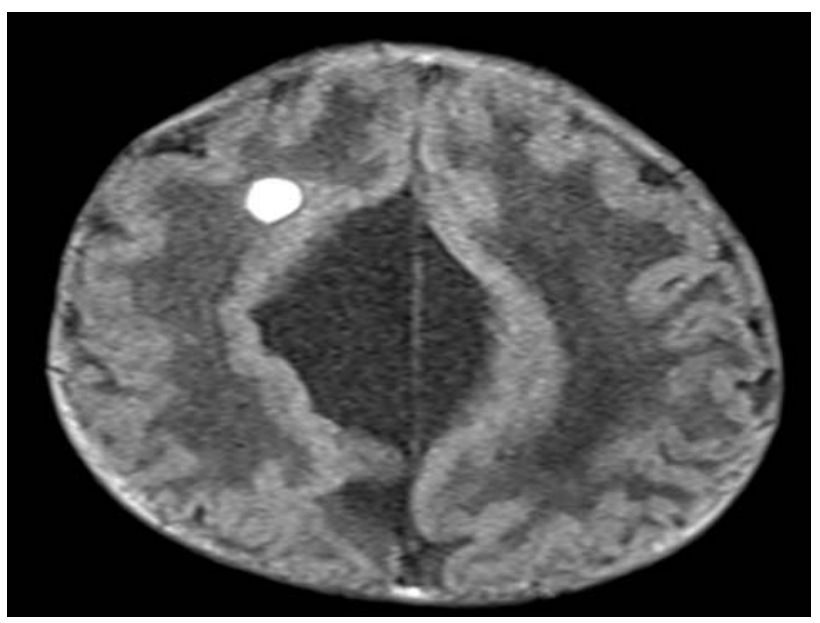

Fig. 4. There is early subacute hematoma at right cerebral hemisphere on T1-weighted axial image. In addition, an interhemispheric cyst $(3.5 \mathrm{~cm}$ in size) is seen at largest area of interhemispheric region.

were observed on brain MRI. Also, there was early subacute hematom at right cerebral hemisphere. In addition, a cyst $(3.5 \mathrm{~cm}$ in size) was observed at interhemispheric region (Figure 4). It was failed to observe septum pellucidum. The patient was referred to neurosurgery department where ventriculoperitoneal shunt was inserted. Electromyography was found to be consistent with myopathy.

The patient who had MRI findings, marked elevation of CK and EMG results compatible with myopathy presented a severe clinical manifestation. Eye and brain findings together 
with above-mentioned findings suggested an intermediate form of congenital muscular dystrophy-dystroglycanopathy with brain and eye anomalies between Walker Warburg syndrome and muscle-eye-brain disease.

\section{Discussion}

In the case discussed, there was hydrocephaly and multiple brain anomalies, cataract, enophthalmia, elevated creatine kinase level and myopathy as demonstrated by electromyography. The patient who had parents with consanguinity (first degree cousins) was consistent with disorders in clinical spectrum of congenital muscular dystrophydystroglycanopathy with brain and eye anomalies which is inherited in autosomal recessive manner. The diagnostic criteria for WWS having more severe clinical course include type 2 lissencephaly, cerebellar malformation, retinal malformation and congenital muscular dystrophy (1). Crowe et al. (3) accepted association of congenital hydrocephaly, bilateral microphthalmia, severe development retardation and multiple brain malformations as diagnostic criteria for WWS. In addition, myopathy is one of the most emphasized diagnostic criteria (4). Dobyns et al. (1) have identified as characteristics in the WWS diagnosis of the anterior or posterior eye anomalies and mentioned as less accompanying eye symptoms of the other eye symptoms such as microphtalmia.

In our case, there were enophthalmia, congenital cataract and posterior synechiae in ophthalmological examination. Congenital glaucoma, progressive myopia, retinal atrophy and juvenile cataract are frequently seen in MEB disease (2). In a study on 7 patients and 8 fetuses with WWS from 8 families, it was reported that most common ocular findings included microphthalmia, buphthalmos, congenital glaucoma, congenital cataract, corneal clouding, anterior chamber dysgenesis, and retinal dysplasia (5). Posterior synechiae is one of the specific findings for the WWS (1) and also cataract has been reported in many cases with WWS $(6,7)$. Eye findings of our patient support the possibility of a diagnosis of WWS. However, enophthalmia hasn't been reported in both the WWS and MEB so far. So, enophthalmia could be a new finding for the diseases in WWS and MEB spectrum.

The diagnostic brain MRI findings include hydrocephaly, frontal bossing, ventriculomegaly, cobblestone lissencephaly with agyria and corpus callosum agenesis, hypoplasia cerebellar vermis, encephalocele and pachygyria-agyria complex. All frequently seen brain anomalies other than cobblestone lissencephaly and encephalocele were present in our case. It has been emphasized that type 2 lissencephaly is the characteristic feature that must be present in WWS (1). Although presence of pachygyria-agyria complex suggested WWS in our case, absence of type 2 lissencephaly made the diagnosis of WWS unlikely.

As in our case, agenesis of the corpus callosum was previously reported in the children with WWS of a Turkish family (8). MRI findings include pachygyria with preferential frontoparietal involvement, polymicrogyria, cerebellar hypoplasia or dysplasia, and flattening of the pons and brainstem associated with eye abnormalities in MEB disease which is defined as a congenital muscular dystrophy with less severe brain anomalies relative to WWS $(2,9)$. Brainstem hypoplasia can be seen in both WWS and MEB disease. In our case, hypoplasia of cerebellum and brainstem was prominent. There was pontine and cerebellar hypoplasia in a case with MEB reported from Pakistan in 2007. Cerebellar cysts have also been reported in MEB, but interhemispheric cyst and agenesis of septum pellucidum seen in our case has never been reported in any form of congenital muscular dystrophy-dystroglycanopathy with brain and eye anomalies. Therefore, these findings may be two new findings accompanying with the diseases of the congenital muscular dystrophy-dystroglycanopathy with brain and anomalies spectrum. Cerebral hematoma seen in our patient has not been reported previously, the cerebral hematoma can either be an association or just a new coincidence.

Although absence of encephalocele, type 2 lissencephaly as well as microphthalmia suggested that the case was consistent with muscle-eye-brain disease rather than WWS, presence of corpus callosum anomaly, congenital contracture and seizure with severe clinical findings suggest that it can be an intermediate form between WWS and MEB.

We presented this case having characteristic features of congenital muscular dystrophydystroglycanopathy with brain and eye anomalies in order to emphasize features that have never been identified in these cases such as enophthalmia, interhemispheric cyst and cerebral hematoma. 


\section{References}

1. Dobyns WB, Pagon RA, Armstrong D, et al. Diagnostic criteria for Walker-Warburg syndrome. Am J Med Genet 1989; 32: 195-210.

2. Godfrey C, Clement E, Mein R, et al. Refining genotype phenotype correlations in muscular dystrophies with defective glycosylation of dystroglycan. Brain 2007; 130: 2725-2735.

3. Crowe C, Jassani M, Dickerman L. The prenatal diagnosis of Warburg syndrome. (Abstract) Am J Hum Genet 1985; 37: 214.

4. Greenberg CR, Jacobs HK, Nylen TE, et al. Congenital hydrocephalus secondary to WalkerWarburg syndrome identified on the Manitoba Neonatal Screening Programme for Duchenne muscular dystrophy. J Med Genet 1992; 29: 583-585.

5. Cormand B, Pihko H, Bayés M, et al. Clinical and genetic distinction between Walker-Warburg syndrome and muscle-eye-brain disease. Neurology 2001; 56: 1059-1069.

6. Whitley CB, Thompson TR, Mastri AR, Gorlin RJ. Warburg syndrome: lethal neurodysplasia with autosomal recessive inheritance. J Pediatr 1983; 102: 547-551.

7. Towfighi J, Sassani JW, Suzuki K, Ladda RL. Cerebro-ocular dysplasia-muscular dystrophy (CODMD) syndrome. Acta Neuropathol 1984; 65: 110-123.

8. Beltrán-Valero de Bernabé D, Currier S, Steinbrecher $\mathrm{A}$, et al. Mutations in the O-mannosyltransferase gene POMT1 give rise to the severe neuronal migration disorder Walker-Warburg syndrome. Am J Hum Genet 2002; 71: 1033-1043.

9. Mercuri E, Messina S, Bruno C, et al. Congenital muscular dystrophies with defective glycosylation of dystroglycan: a population study. Neurology 2009; 72: 1802-1809. 